\title{
Vancomycin Resistant Enterococcus
}

National Cancer Institute

\section{Source}

National Cancer Institute. Vancomycin Resistant Enterococcus. NCI Thesaurus. Code C76312.

A non-taxonomic grouping of enterococcus species that are resistant to vancomycin antibiotics through alteration of the peptidoglycan synthesis pathway. 